\title{
Correction of Hypoxia-Induced Disorders in Nephrotic Children
}

\author{
Maidannyk V, Burlaka Ie \\ O.O. Bogomolets National Medical University, Department of Pediatrics №4 \\ Kyiv, Ukraine, Tolstogo Street 10, 01004 \\ maidannyk@gmail.com \\ evgbur1982@gmail.com
}

\begin{abstract}
:
Objectives: the objective of this paper was to study the levels of cellular hypoxia, apoptosis controlling factors in relation to the value of kidney function impairment and to evaluate possible protective effects of the treatment with antioxidant agent tocopherol in pediatric patients with nephrotic syndrome.

Background: hypoxia-related disorders play an important role in irreversible tissue damage via activation of certain secondary processes.

Methods: an examination of renal biopsies of 53 patients (aged 10 to 15 years) with nephrotic syndrome was done. Conventional clinical investigations, immunogistichemistry, immunoblotting were used in this study.

Results: we show that nephrotic patients reveal a high level of marker of cellular hypoxia HIF-1 $\alpha$ and its dependence on the level of kidney function impairment and proteinuria. The progression of the sclerosis as a sign of irreversible kidney damage is accompanied by gradual increase in expression of proapoptotic factor Bax. An applied treatment composes conventional scheme and tocopherol showed an improvement in hypoxia level, proapoptotic factor Bax expression.
\end{abstract}

Conclusion: hypoxia-induces disorders in nephrotic children presented by disturbances in apoptosis controlling system can be attenuated by the antioxidants application.

Keywords: treatment, tocopherol, nephrotic syndrome, HIF-1 $\alpha$, Bax, immunostaining.

This work was covered by the grand $0105 \mathrm{U} 003769$.

\section{INTRODUCTION}

The incidence of children with chronic kidney disease (CKD) is from 1.5 to 3.0 cases per million of population. The main causes of CKD in childhood are developmental abnormalities (congenital anomalies of the kidney and urinary tract), focal segmental glomerulosclerosis (FSGS), hemolytic uremic syndrome (HUS), immune-complex diseases, inherited abnormalities, Alport syndrome etc [1,2]. Main pathomorphological outcomes that apply to kidney damage in CKD are glomerulosclerosis, vascular sclerosis, tubule-interstitial fibrosis. Adaptive changes of nephrons after the primary injury which can no longer be compensated with time, ultimately lead to irreversible disorders - scarring, sclerosis and further loss of nephrons leading to the end-stage CKD (ES-CKD) formation [2]. Inflammation plays an important role in the development and progression of chronic kidney pathologies and is the primary and persistent violation, which underlies the pathogenesis of others. Renal histology in chronic renal pathologies including nephrotic syndrome is characterized by typical signs of inflammation i.e. infiltration with white blood cells, hyperemia, fibrosis etc. In addition to inflammation, fibrosis has a role in nephrotic syndrome. These disorders are accompanied by activation of the renin-angiotensin-aldosterone system, oxidative stress, endothelial dysfunction and others [1-3]. All mentioned above pathophysiological violations might be acompanied by apoptosis. Apoptosis is programmed cell death that occurs when kidney disease has a place and plays an important role in their physiology. Harmful effects of apoptosis are in fact a source of a large number of kidney cells lost during and/or renal inflammation, scarring, loss of kidney function [4,5] The molecular mechanisms 
underlying irreversible renal damage in children with nephrotic syndrome depending on apoptosis activation might be a potential therapeutic issue in CKD treatment.

The main tasks in CKD treatment are renal insufficiency compensation, complications prevention and lowering the rate of 6he disease progression. Basic medicines for CKD treatment are immunoregulative drugs (corticosteroids, immunosuppressors), ACEi, Angiotensin receptors blockers. The last two groups provide protective effect through slowing down proteinuria and blood pressure lowering in nephron [6]. However, there are no specific non-toxic drugs regulating apoptosis and apoptosis-dependent disorders in this cohort of patients.

\section{Materials AND Methods}

\subsection{Patients}

An examination of renal biopsies of 53 patients (aged 10 to 15 years) with nephrotic syndrome hospitalized in Pediatric Nephrology unit of the Children Clinical Hospital №7 (Kyiv, Ukraine) was done. All patients were treated accordingly the local protocols. Among all patients 24 $(45,28 \%)$ were with hormone-sensitive type of nephrotic syndrome, others - $29(54,72 \%)$ showed hormone-dependent type of nephrotic syndrome. Complex examination other than conventional methods (inspection, monitoring blood pressure, general and biochemical blood tests, determination of daily proteinuria, urinary sediment study and concentration ability of the kidneys, ultrasound of the abdomen etc.), immunohistochemical assessment of apoptosisdependent glomerular and tubule-interstitial damage were done.

The level of kidney function impairment (stage of Chronic Kidney Disease, CKD) was assessed by the value of glomerular filtration rate (GFR). GFR was calculated by Schwartz formula.

All patients were treated accordingly to local protocols which include corticosteroids (prednisolone), immunosuppressive drugs (CellCept, Leikeran), ACEi, diuretics (furosemide). An antioxidant (Vitamin E - tocopherol) beside mentioned above drugs in dose $100 \mathrm{mg} /$ day was administered to patients from experimental group for 1 month.

\subsection{Immunohistochemistry}

Renal tissue (3- $\mu \mathrm{m}$ sections) was deparaffinized and rehydrated prior to processing. Antigen retrieval was carried out by boiling in Citrate Buffer $(10 \mathrm{mM}$ Citric Acid, $0.05 \%$ Tween20, $\mathrm{pH}$ 6.0) for $20 \mathrm{~min}$. Sections were treated with Triton X-100 0.3\% (Sigma-Aldrich NV/SA, Bornem, Belgium) in PBS for 20min. After three PBS washes, sections were incubated with blocking buffer (5\% bovine serum albumin and Triton X-100 0.1\% in PBS) for 1 hour. The rabbit polyclonal anti-Bax antibody (Santa Cruz, CA, USA) was applied at a dilution of 1:50 in 5\% bovine serum albumin in PBS overnight at $4 \mathrm{C}$. Following three PBS washes, sections were incubated with a secondary Alexa Fluor 488 goat anti-rabbit IgG (1:500) for $1 \mathrm{~h}$ at room temperature. Nuclei were counterstained with DAPI. All samples were stained for an identical length of time and under identical conditions. All recordings for each staining were done during one day using identical gain settings. Sections were mounted in Immu-Mount (Thermo Shandon, Midland, Canada) and observed with Leica TCS SP inverted confocal scanning laser microscope using $25 \mathrm{X} / 0.8 \mathrm{NA}$ oil-immersion objective. Image analysis was performed using ImageJ software (NIH Image, Baltimore, MD, USA). Three areas in each section were analyzed.

\subsection{Immunoblotting for detection of Bax, HIF-1 $\alpha$}

Proteins solubilized in Laemmli sample buffer were resolved in polyacrylamide gels by SDSPAGE and transferred to a polyvinylidene difluoride membrane. Membranes were then blocked in $5 \%$ non-fat milk in TBS-T (136 mM NaCl, $10 \mathrm{mM}$ Tris, $0.05 \%$ Tween 20$)$ and immunoblotted using the Bax and HIF-1 $\alpha$ Ab (Cell Signaling Technology, Danvers, MA USA) and actin mouse $\mathrm{mAb}$ (BD, Lexington KY, USA) for $1 \mathrm{~h}$ at room temperature. The actin mouse $\mathrm{mAb}$ were used as a loading control. After three washes with TBS-T, the membranes were incubated with secondary anti-rabbit or anti-mouse antibodies labeled with horseradish peroxidase for $1 \mathrm{~h}$ at room temperature. Membranes were washed three times with TBS-T. The protein bands were visualized by chemiluminescent substrate ECL. Quantitation of the protein content was done by densitometric analysis. 


\section{Statistics}

Statistical analysis was done using the method of variation statistics (STATISTICA 6.0) and nonparametric statistical approaches (Mann-Whitney test). Results are presented as Mean \pm SEM. $\mathrm{P}<0.05$ was considered as statistically significant.

\section{RESUlts}

We have detected an increased level of marker of cellular hypoxia (HIF-1 $\alpha$ ) in blood serum of all patients with nephrotic syndrome as compared to control group. Moreover, a dependence between the levels of renal insufficiency (assessed by GFR) and cellular hypoxia has been documented. The levels of HIF- $1 \alpha$ in group with CKD I st. was detected at level over excided the control group value by $28,6 \%(\mathrm{P}<0.01$ compared to control group) and by $41,3 \%$ ( $\mathrm{p}<0,01$ compared to control group) in patients with CKD II-III st. (Figure 1).

A.

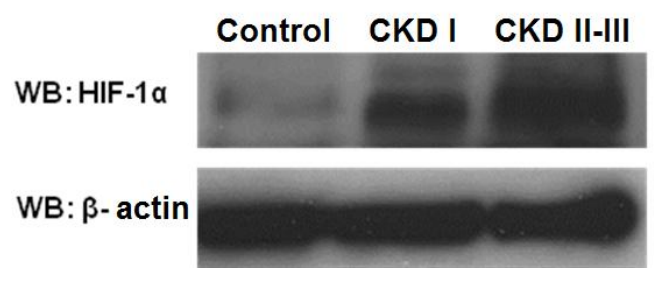

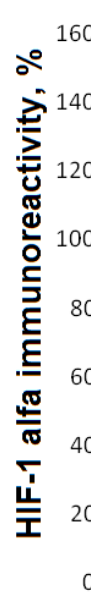

B.

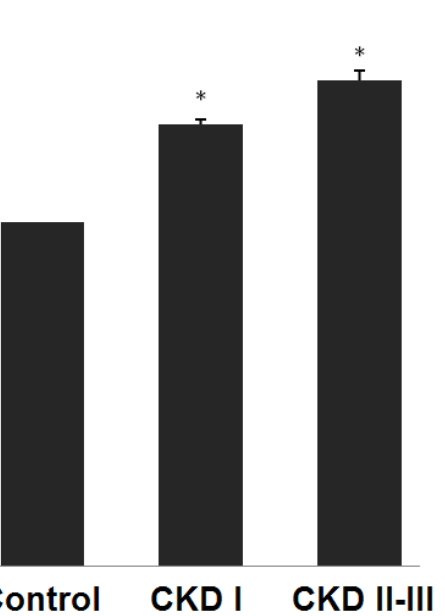

Figure 1. Level of HIF-1 $\alpha$ in plasma of patients with nephritic syndrome and control. A - level HIF-1 $\alpha$; $B$ HIF-1 $\alpha$ immunoreactivity; * - $p^{<} 0,05$. WB - Western Blotting.

Individual analysis of the HIF-1 $\alpha$ levels in serum and correspondent levels of daily proteinuria in patients with nephritic syndrome identified a direct positive correlation $(r=0,61, p<0,01)$ (Figure 2).

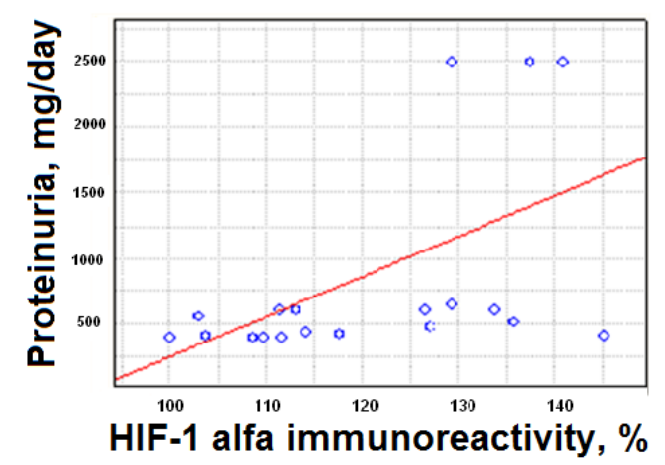

Figure 2. Dependence of HIF-1 $\alpha$ levels on daily proteinuria values in children with nephritic syndrome.

These data indicate the dependence of chronic hypoxia on the degree of damage to the kidney filtration barrier and is a proof of the direct influence of hypoxia and CKD progression.

\section{Expression of proapoptotic factor Bax in kidney tissue of patients with nephrotic syndrome}

We have analyzed the levels of expression and localization of proapoptotic factor Bax in patients with morphological variant of nephrotic syndrome - focal segmental glomerulosclerosis. Stages of FSGS were determined by level of glomerular sclerotic area. Level of sclerosis corresponding to $\leq 25 \%$ of the glomerular area was assumed as I stage of FSGS, II stage of FSGS - 25-50\%, III stage - 50-75\% and IV stage - 75-100\%. Analysis of Bax expression in kidney biopsies from children with focal segmental glomerulosclerosis show the presence of high level of Bax expression in both glomerular and tubule-interstitial segments. Higher level of immune signal was 
recorded in glomeruli as compared to tubule-interstitial segment in FSGS I-II stages $(43,57 \pm 0,88$ a.u. vs $24,9 \pm 0,41$ a.u., $\mathrm{P}<0.01)$. When complete glomerular sclerosis presents a high level of $\mathrm{Bax}$ was documented in the surrounding tubule-interstitial segment $(13,7 \pm 0,42$ a.u. vs $22,5 \pm 0,65$ a.u., P<0.01) (Figure 3).
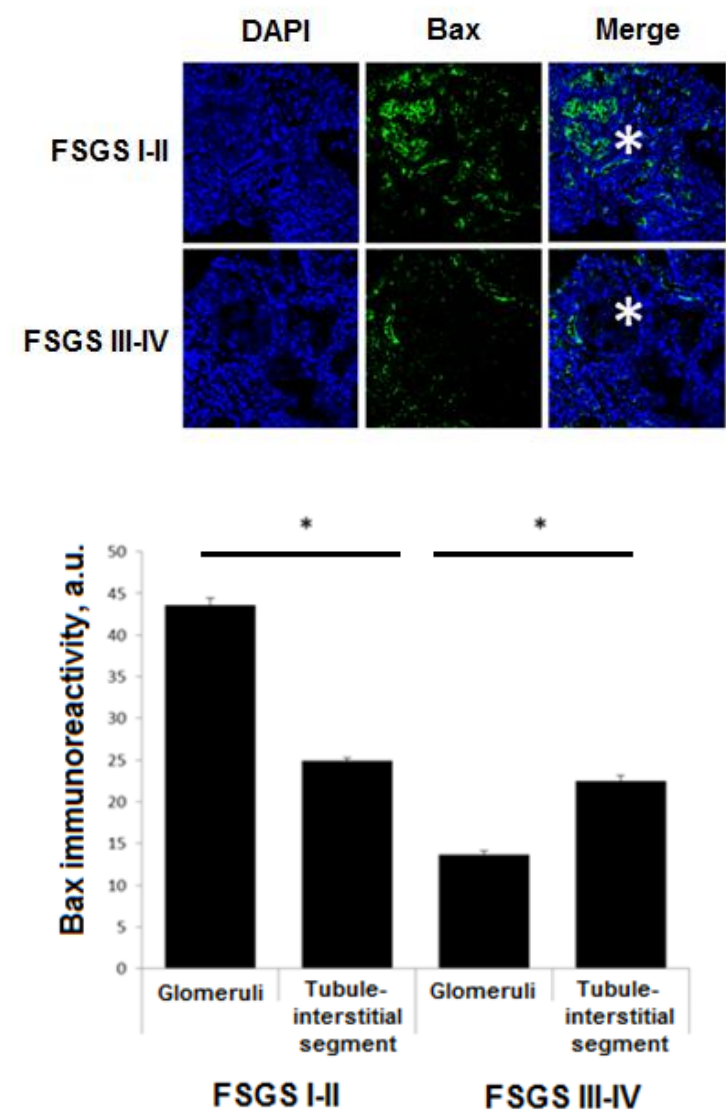

Figure 3. Topical characteristic of the Bax expression in different stages of FSGS. DAPI - visualization of nuclei; Bax - Bax immune signal in kidney tissue. * - glomeruli, * $p^{<} 0,05$.

\section{Therapeutic effects on hypoxia-induced damages in patients with nephrotic syndrome}

To evaluate the effectiveness of treatment of children with nephritic syndrome all patients were randomized and divided into three groups. The first group (main group) were children with nephrotic syndrome who received conventional basic therapy. The second (experimental group) were children with nephrotic syndrome who received conventional basic therapy and tocopherol.The third group (control) included 45 healthy children matched by age and sex.

After a course of therapy all children were investigated for serum level marker of chronic hypoxia HIF- $1 \alpha$. In children from from main group the level of HIF-1 $\alpha$ exceeded indicator of the control group by $26,23 \pm 2,2 \%$ (p <0.001). After a course of treatment the level HIF-1 $\alpha$ in this group exceeded control rate by $18,56 \pm 2,49 \%$ ( $\mathrm{p}<0.01$ in comparison to the level before treatment).Children from the experimental group showed a level of HIF-1 $\alpha$ exceeded the control group by $24,9 \pm 1,76 \%$ ( $\mathrm{p}<0.001$ ). After the course of treatment HIF-1 $\alpha$ was decreased almost to the level of control group (Figure 4).

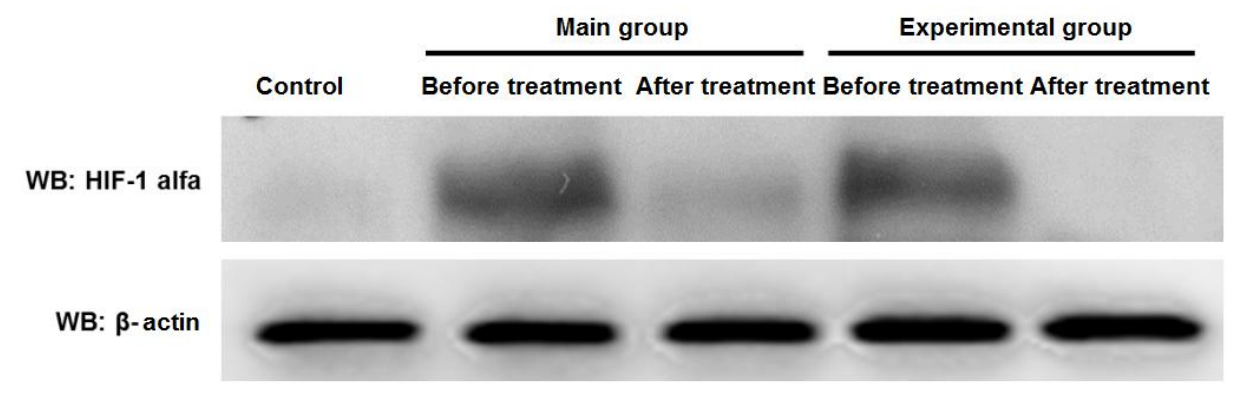




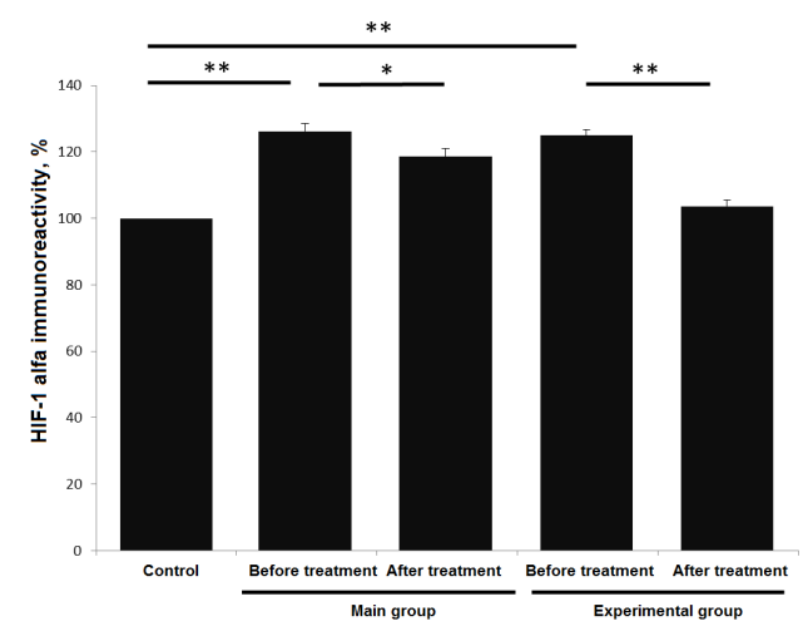

Figure 4. Levels of hypoxia HIF-1 $\alpha$ in children with nephrotic syndrome before and after the treatment. Note: A - level HIF-1 $\alpha$; B - immunoreactivity; * $P<0.01$, ** $-P<0.001$.

After the course of therapy all examined children were investigated for serum levels of proapoptotic factor Bax. In children included to the main group Bax concentration was increased up to $124,7 \pm 3,45 \%$ ( $\mathrm{p}<0.001$ ) as compared to control. Value of control control group was taken as $100 \%$. After a course of treatment Bax value was decreased to $112,3 \pm 1,6 \%(\mathrm{p}<0.05)$ as compared to level before treatment. Children from experimental group exhibited Bax before treatment at level $125,5 \pm 1,9 \%(\mathrm{p}<0.001)$ as compared to control group. After a course of treatment Bax was down regulated to $105,2 \pm 2,02 \%(\mathrm{p}<0.01$ in comparison to the level before treatment) (Figure 5).
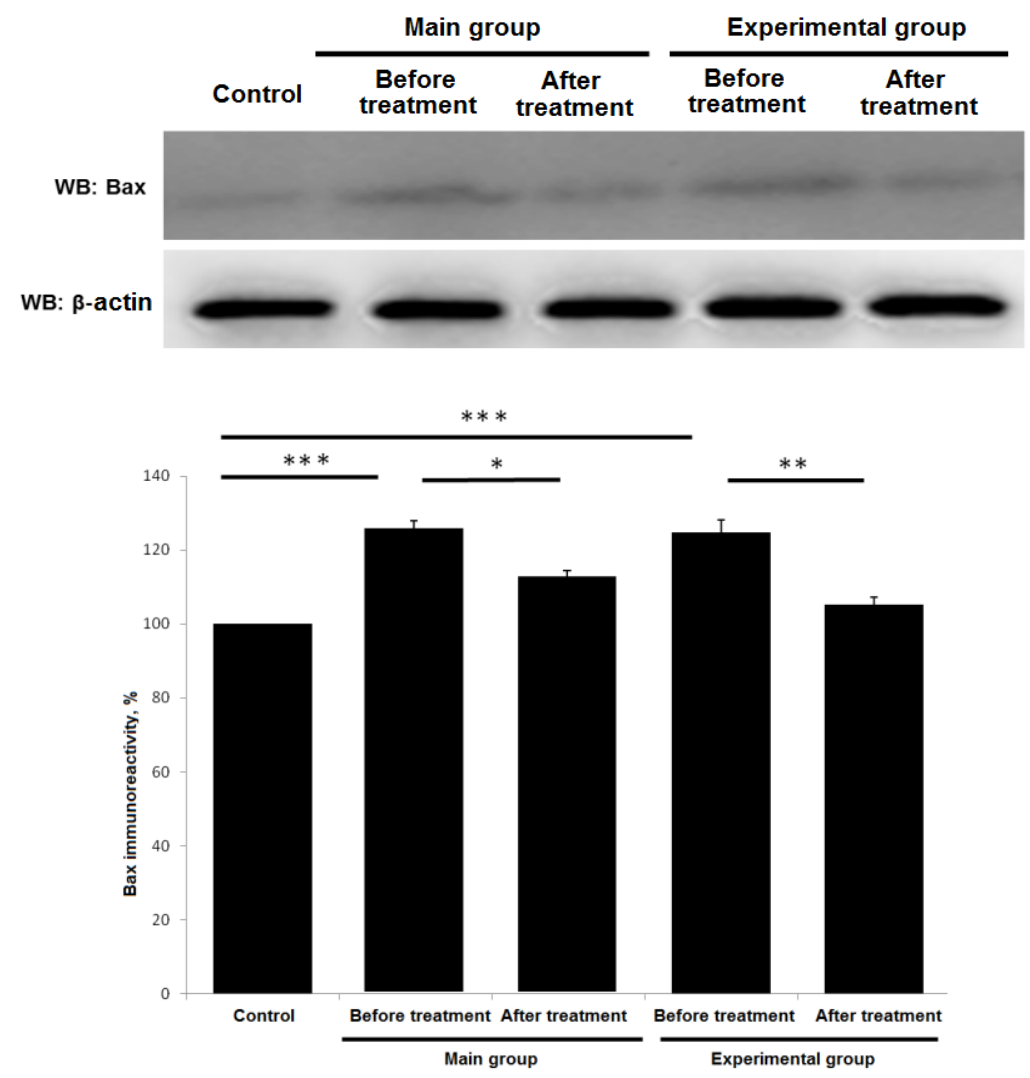

Fig. 5. Levels of proapoptotic factor Bax in children with nephritic syndrome before and after the treatment. A - level of Bax; $B$ - immunoreactivity; * $P<0.05$, ** $-P<0.01$, *** - $P<0.001$.

\section{DISCUSSION}

Tissue hypoxia is a pathologic feature of many human diseases including those associated with inflammation. The hypoxia-inducible factors (HIFs) comprise a family of oxygen-sensitive basic 
helix-loop-helix proteins that control cellular transcriptional response to hypoxia [7]. It was shown that HIF signaling could potentially promote the development of fibrosis particularly due its role in inflammation $[7,8]$.

Pathogenesis of the nephrotic syndrome deals with inflammation leading to progression and development of the irreversible tissue damages. Hypoxia is an important link in inflammation development. A mechanism by which HIF may impact the pathogenesis of kidney damage is through the regulation of inflammatory responses. Microenvironmental changes, such as hypoxia, strongly impact inflammatory cell recruitment and function [8]. Moreover, hypoxia has been shown to induce apoptosis, where HIF-1 plays a complex role. It has also been demonstrated that the expression of HIF- $1 \alpha$ significantly correlated with apoptosis and the pro-apoptotic factors, such as caspase-3, Fas, and Fas ligand. This finding has been shown on in vitro models [9].

Here we show that both over expression of HIF-1 $\alpha$ and apoapoptosic factor Bax have a place in children with nephritic syndrome meaning that chronic hypoxia is a factor predisposing disturbances in system controlling apoptosis in this cohort of patients. Our data demonstrate that there is a dependence between the level of hypoxia, Bax overexpression and the stage of CKD. We show that progression of glomerulosclerosis in children with nephrotic syndrome is accompanied by increased activity of proapoptotic factor. Revealed dependence of topology of Bax levels on FSGS degree indicates that development of glomerular and tubule-interstitial disorders under the influence of proteinuria occurs in specific range.

Tocopherol belongs to the family members of a fat-soluble antioxidant that is able to intercept free radicals in the plasma membrane, which helps to prevent the oxidative damage to lipids. It is known that patients with CKD have reduced levels of tocopherol in plasma, which is a prerequisite for this use in therapies [10]. Previous studies have shown that administration of tocopherol in patients with CKD helped to reduce the risk of cardiovascular complications, increased activity of endogenous antioxidant systems - GPX, catalase [11]. It has been shown that treatment with an antioxidant lowered the hypoxia-induced cell damage as well as the elevation of HIF-1alpha in vitro [12]. Here we demonstrate that tocopherol administration has a potent effect on hypoxia-induced apoptosis development in children with nephrotic syndrome and subsequent restoration of the proapoptotic factor Bax activation.

Further study of the molecular mechanisms which provides kidney protection in nephrotic patients upon the treatment with antioxidants in addition to the conventional therapy will be done.

\section{ACKNOWLEDGEMENTS}

This work has been done with support from nephrology unit of Children Clinical Hospital №7 (Kyiv, Ukraine).

\section{REFERENCES}

[1]. Fogo A.B. Mechanisms of progression of chronic kidney disease. Pediatr Nephrol. - 2007. 22 (12): 2011-2022.

[2]. Hemmelgarn B.R., Manns B.J., Lloyd A., James M.T., Klarenbach S. Relation between kidney function, proteinuria, and adverse outcomes 2010; JAMA 303: 423-429.

[3]. Favaloro B., Allocati N., Graziano V., Di Ilio C., De Laurenzi V. Role of apoptosis in disease. Aging 2012; 4 (5): 330-349.

[4]. Christman J.W., Blackwell T.S., Juurlink B.H.J. Redox Regulation of Nuclear Factor Kappa B: Therapeutic Potential for Attenuating Inflammatory Responses. Brain Pathology 2000; 10: $153-162$.

[5]. Abbate M., Zoja C., Remuzzi G. How does proteinuria cause progressive renal damage? J Am Soc Nephrol 2006; 17: 2974-2984.

[6]. KDIGO 2012 Clinical Practice Guideline for the Evaluation and Management of Chronic Kidney Disease. Kidney Int Suppl 2013; 3:5.

[7]. Bento C.F., Pereira P. Regulation of hypoxia-inducible factor 1 and the loss of the cellular response to hypoxia in diabetes. Diabetologia 2011; 54:1946-1956.

[8]. Eltzschig H.K., Carmeliet P. Hypoxia and inflammation. N Engl J Med 2011; 364(7): 656665 . 
[9]. Volm M, Koomägi R. Hypoxia-inducible factor (HIF-1) and its relationship to apoptosis and proliferation in lung cancer. Anticancer Res 2000; 20(3A):1527-1533.

[10]. Bin Q., Hu X., Cao Y., Gao F. The role of vitamin E (tocopherol) supplementation in the prevention of stroke. A meta-analysis of 13 randomized controlled trials 2011; Thrombosis and haemostasis 105 (4): 579-585.

[11]. Young J.F., Rosenvold K., Stagsted J., Nielsen J.H., Andersen H.J. Significance of vitamin E supplementation, dietary content of polyunsaturated fatty acids, and preslaughter stress on oxidative status in pig as reflected in cell integrity and antioxidative enzyme activities in porcine muscle. J Agric Food Chem 2005; 53(3): 745-749.

[12]. Magalhães J., Ferreira R., Neuparth M.J., Oliveira P.J., Marques F., Ascensão A. Vitamin E prevents hypobaric hypoxia-induced mitochondrial dysfunction in skeletal muscle. Clin Sci (Lond) 2007; 113(12): 459-466. 